\title{
Global Asymptotic Stabilization of a Spinning Top With Torque Actuators Using Stereographic Projection
}

\author{
C. J. WAN \\ Department of Aerospace Engineering, The University of Michigan, Ann Arbor, MI 48109-2140, USA \\ P. TSIOTRAS \\ School of Aeronautics and Astronautics, Purdue University, West Lafayette, IN 47907-1282, USA; currently, Dept. \\ of Mechanical, Aerospace and Nuclear Engineering, University of Virginia, Charlottesville, VA 22903-2242, USA \\ V. T. COPPOLA \\ Department of Aerospace Engineering, The University of Michigan, Ann Arbor, MI 48109-2140, USA \\ D. S. BERNSTEIN \\ Department of Aerospace Engineering, The University of Michigan, Ann Arbor, MI 48109-2140, USA
}

Editor: M. J. Corless

Received January 3, 1994; Revised January 24, 1996

\begin{abstract}
The dynamical equations for a spinning top are derived in which the orientation is specified by a complex variable using stereographic projection of Poisson's equations. Necessary and sufficient conditions for Lyapunov stability of the uncontrolled motion of the spinning top are derived using the Energy-Casimir method. Control laws that globally asymptotically stabilize the spinning top to the sleeping motion using two torque actuators are synthesized by employing techniques from the theory of systems in cascade form and generalized using Hamilton-Jacobi-Bellman theory with zero dynamics. In short, this paper provides a nice example where the interplay between dynamics and control leads to elegant and powerful results.
\end{abstract}

Keywords: spinning top, stabilization, stereographic projection, Lyapunov function, Hamilton-Jacobi theory

\section{Introduction}

In this paper we examine the problem of the global asymptotic stabilization of a spinning top with fixed vertex, to a uniform, steady rotation about its axis of symmetry. This motion of the top is often referred to in the literature as the sleeping top [7]. This terminology arises because a smooth, axially symmetric top with its symmetry axis vertical, might appear at first glance to be not moving at all, and hence "sleeping." Stability analysis of the sleeping motion of a spinning top is well-developed. In [22], the authors summarized the previous results and gave necessary and sufficient conditions for the Lyapunov stability of the sleeping motion which simplified the earlier results given by $\mathrm{Ge}$ and $\mathrm{Wu}$ [6].

The controlled top problem, i.e., applying control inputs to drive the spinning top to the sleeping motion, was studied in [22]. In [22], the control inputs are inertially-fixed horizontal forces and the kinematic formulation was based on the 2-1-3 Euler angles. Asymptotically stabilizing control laws were derived using the feedback linearization and 
the Hamilton-Jacobi-Bellman theory with zero dynamics for the case of two control forces. In the case of only one control force, if the top is spinning sufficiently fast, asymptotically stabilizing control laws were developed by the Jurdjevic-Quinn technique.

In this paper, we consider another controlled top problem which uses alternative control inputs, namely, body-fixed torques. It is well-known that the sleeping motion of a spinning top is Lyapunov stable if its spin rate is sufficiently high [4], [6], [7], [11], [12], [22]. We give the necessary and sufficient condition for stability about the vertical of an uncontrolled spinning top using the Energy-Casimir method [12], [21]. This condition coincides with the previous results [6], [22] and implies that the spinning top can be Lyapunov stable, but a minimum amount of spin rate is necessary in order to achieve Lyapunov stability for the sleeping motion, when no other control input is available. Here we remove this restriction and consider stabilization without any requirement on the magnitude of the spin rate. Moreover, the results hold also for the extreme case when the spin rate remains zero. Two control torques about the top's transverse principal axes are used in order to achieve this.

The formulation of the problem departs from the traditional treatment-based on Eulerian angles - and takes advantage of a new formulation for the kinematics of the rotational motion developed in [14]. This new kinematic formulation uses the stereographic projection of the Riemann sphere on the complex plane in order to derive a very elegant and compact equation for a complex quantity related to the direction cosines of the local vertical (the inertial $Z$-axis) with respect to the local body-fixed system of the top. This kinematic formulation is based on an idea by Darboux [5], where an equation of the same form was derived in connection with some problems in classical differential geometry. However, its derivation using the stereographic projection and its use in attitude kinematics was established in [14] and was first applied to attitude control problems of spinning rigid bodies in [15], [16], [17], [18].

The paper is organized as follows. In Section 2 we give the equations of motion of a spinning top rotating in a uniform gravitational field and we derive the corresponding equations using complex variables from the stereographic projection of the coordinates of Poisson's equations. In Section 3 we examine the stability of the free motion of the top in terms of the complex formulation and we provide necessary and sufficient conditions for (nonlinear) Lyapunov stability using the Energy-Casimir method. In Section 4 we derive globally asymptotically stabilizing feedback control laws for uniform rotation of the top along the local vertical. We first give a list of control laws based on the stereographic coordinates. In the last part of Section 4 we use some recent results from the theory of optimal asymptotic stabilization of nonlinear systems with stable zero dynamics based on Hamilton-Jacobi-Bellman theory [19], [20]. We therefore generalize the previous stabilizing control laws by constructing a family of optimal nonlinear feedback control laws which globally asymptotically stabilize the spinning top. The parameters of this family can then be tuned by the designer to achieve acceptable system performance (minimum control effort, time of response, etc.). Finally, a numerical example demonstrates the results of the proposed control laws. 


\section{Equations for a Spinning Top Using Stereographic Projection}

In this section we derive the dynamical equations of a spinning top using stereographic projection of Poisson's equations [14], [15]. Traditionally, the motion of an uncontrolled spinning top is described by the Euler-Poisson system of equations [11], [22] given by

$$
\begin{aligned}
& J_{1} \dot{\omega}_{1}=\left(J_{2}-J_{3}\right) \omega_{2} \omega_{3}+m g \ell \gamma_{2} \\
& J_{2} \dot{\omega}_{2}=\left(J_{3}-J_{1}\right) \omega_{3} \omega_{1}-m g \ell \gamma_{1} \\
& J_{3} \dot{\omega}_{3}=\left(J_{1}-J_{2}\right) \omega_{1} \omega_{2}
\end{aligned}
$$

and

$$
\begin{aligned}
& \dot{\gamma}_{1}=\gamma_{2} \omega_{3}-\gamma_{3} \omega_{2} \\
& \dot{\gamma}_{2}=\gamma_{3} \omega_{1}-\gamma_{1} \omega_{3} \\
& \dot{\gamma}_{3}=\gamma_{1} \omega_{2}-\gamma_{2} \omega_{1} .
\end{aligned}
$$

Equations (1) describe the dynamics of the motion with respect to a body-fixed reference frame located at the vertex of the top and equations (2) describe the kinematics. In (1) and (2) $\omega_{1}, \omega_{2}, \omega_{3}$ are the angular velocity vector components in body coordinates, $m$ is the mass of the top, $g$ is the gravitational constant and $\ell$ is the distance from the vertex to the center of mass. The parameters $J_{1}, J_{2}, J_{3}$ represent the principal moments of inertia with respect to the chosen body-fixed reference frame. The vector $\left(\gamma_{1}, \gamma_{2}, \gamma_{3}\right)$ represents the unit vector in the negative gravity direction when expressed in body coordinates. In other words, $\gamma_{1}, \gamma_{2}, \gamma_{3}$ are the direction cosines of the inertial $Z$-axis (considered here to point along the negative gravity direction) with respect to the local body-fixed axes. Therefore, equations (2) actually describe the tilt angles of the body axes of the top from the inertial $Z$-axis, while the azimuth between the projection of the top axis on the horizontal plane and any axis fixed in the horizontal plane is not determined.

We assume that the top is symmetric, i.e., $J_{1}=J_{2} \triangleq J$, and therefore from (1c) we find that $\omega_{3}$ is constant. Define $\omega_{3} \triangleq \Omega, b \triangleq J_{3} \Omega / J$ and $c \triangleq 2 m g \ell / J$. Then the equations (1) and (2) can be written in the form

$$
\begin{aligned}
& \dot{\omega}_{1}=-(b-\Omega) \omega_{2}+\frac{c}{2} \gamma_{2} \\
& \dot{\omega}_{2}=(b-\Omega) \omega_{1}-\frac{c}{2} \gamma_{1}
\end{aligned}
$$

and

$$
\begin{aligned}
& \dot{\gamma}_{1}=\gamma_{2} \Omega-\gamma_{3} \omega_{2} \\
& \dot{\gamma}_{2}=\gamma_{3} \omega_{1}-\gamma_{1} \Omega \\
& \dot{\gamma}_{3}=\gamma_{1} \omega_{2}-\gamma_{2} \omega_{1} .
\end{aligned}
$$

We now introduce a reformulation of the kinematics that will simplify the ensuing analysis significantly. This new formulation is based on an idea by Darboux [5], and was initially 
applied to the problem of attitude dynamics in [14], although it appears that Leimanis [11] was also aware of this possibility. Because of the constraint $\gamma_{1}^{2}+\gamma_{2}^{2}+\gamma_{3}^{2}=1$ the vector $\left(\gamma_{1}, \gamma_{2}, \gamma_{3}\right)$ lies on the unit sphere $S^{2}$ in $\mathbb{R}^{3}$. If we consider the stereographic projection $S^{2} \rightarrow \mathbb{C}_{\infty}$ of the unit sphere $S^{2}$ onto the extended complex plane $\mathbb{C}_{\infty} \triangleq \mathbb{C} \cup\{\infty\}$, defined by

$$
\eta=\eta_{1}+i \eta_{2} \triangleq \frac{\gamma_{2}-i \gamma_{1}}{1+\gamma_{3}}=\frac{1-\gamma_{3}}{\gamma_{2}+i \gamma_{1}}
$$

where $i=\sqrt{-1}$, we induce the following differential equation for the complex variable $\eta \in \mathbb{C}[14],[15]$,

$$
\dot{\eta}=-i \Omega \eta+\frac{\omega}{2}+\frac{\bar{\omega}}{2} \eta^{2}
$$

where the bar denotes complex conjugate and where $\omega \triangleq \omega_{1}+i \omega_{2}$. The stereographic projection establishes a one-to-one correspondence between the unit sphere and the extended complex plane. It can be easily verified that the inverse map $\eta \mapsto\left(\gamma_{1}, \gamma_{2}, \gamma_{3}\right)$ is given by

$$
\begin{aligned}
& \gamma_{1}=i \frac{\eta-\bar{\eta}}{1+|\eta|^{2}}=-\frac{2 \eta_{2}}{1+|\eta|^{2}} \\
& \gamma_{2}=\frac{\eta+\bar{\eta}}{1+|\eta|^{2}}=\frac{2 \eta_{1}}{1+|\eta|^{2}} \\
& \gamma_{3}=\frac{1-|\eta|^{2}}{1+|\eta|^{2}}
\end{aligned}
$$

where $|\cdot|$ denotes the absolute value of a complex number, i.e., $z \bar{z}=|z|^{2}, z \in \mathbb{C}$. Using the complex variables $\omega$ and $\eta$, equations (3) and (4) can be expressed compactly as

$$
\begin{aligned}
& \dot{\omega}=i(b-\Omega) \omega+\frac{c \eta}{1+|\eta|^{2}} \\
& \dot{\eta}=-i \Omega \eta+\frac{\omega}{2}+\frac{\bar{\omega}}{2} \eta^{2}
\end{aligned}
$$

and the tilt angle $\Theta$ between the top symmetry axis and the inertial $Z$-axis is

$$
\Theta=\cos ^{-1}\left(\frac{1-|\eta|^{2}}{1+|\eta|^{2}}\right) .
$$

An easy calculation shows that, if $\Omega=b$, i.e., $J_{3}=J$, then the only equilibrium state of equations (8) and (9) is $\omega=\eta=0$. If $\Omega \neq b$, then (apart from the trivial case $\omega=\eta=0$ ) the equilibrium states of the uncontrolled motion of equations (8) and (9) satisfy

$$
|\eta|^{2}=\frac{c-2 \Omega(b-\Omega)}{c+2 \Omega(b-\Omega)}
$$


and

$$
\omega=i \frac{c}{(b-\Omega)} \frac{\eta}{1+|\eta|^{2}}
$$

The expression (11) can be written equivalently as

$$
\gamma_{3}=\frac{2 \Omega(b-\Omega)}{c} .
$$

Since $c>0$, it can be shown that if $b^{2}<2 c$ then $\omega=\eta=0$ is the only equilibrium state. If, on the other hand, $b^{2} \geq 2 c$, then two cases need to be considered, namely, $\Omega>0$ and $\Omega<0$. If $\Omega>0$ and $\frac{1}{2}\left(b+\sqrt{b^{2}-2 c}\right) \geq \Omega>\frac{b}{2}$ or $\Omega \geq \frac{1}{2}\left(b+\sqrt{b^{2}+2 c}\right)$, then $\omega=\eta=0$ is the only equilibrium state. If $\Omega>0$ and $\frac{1}{2}\left(b+\sqrt{b^{2}+2 c}\right)>\Omega>\frac{1}{2}\left(b+\sqrt{b^{2}-2 c}\right)$ and $\Omega \neq b$ then there are nonzero equilibrium states corresponding to the solutions of (11) or (13) and (12). Similarly, if $\Omega<0$ and $\frac{1}{2}\left(b-\sqrt{b^{2}-2 c}\right) \leq \Omega<\frac{b}{2}$ or $\Omega \leq \frac{1}{2}\left(b-\sqrt{b^{2}+2 c}\right)$, then $\omega=\eta=0$ is the only equilibrium state. If $\Omega<0$ and $\frac{1}{2}\left(b-\sqrt{b^{2}+2 c}\right)<\Omega<$ $\frac{1}{2}\left(b-\sqrt{b^{2}-2 c}\right)$ and $\Omega \neq b$ then there are nonzero equilibrium states corresponding to the solutions of (11) or (13) and (12). It is interesting to note that these nonzero equilibrium states correspond to a steady precession of the top. In the steady precession, $|\eta|=$ const . which implies from (10) a constant tilt angle $\Theta$. Note that if $\Omega=0$, then from (8) and (9) one sees that the top degenerates to an inverted spherical pendulum and has only one equilibrium state $\omega=\eta=0$. Note also that the equations for the top reduce to those for a symmetric spacecraft in the case $g=0$ (i.e., $c=0$ ), which has only one equilibrium state, namely, $\omega=\eta=0$. Finally, it should be noted that, because of the well-known properties among the principal moments of inertia, for any physically realizable rigid body, one must have that $\Omega>\frac{b}{2}$ for the case when $\Omega>0$, while if $\Omega<0$, then $\Omega<\frac{b}{2}$.

\section{Stability of the Free Motion of the Spinning Top}

In this section we analyze the (nonlinear) Lyapunov stability of the sleeping motion of the spinning top using Lyapunov's direct method. Stability of the sleeping motion of a spinning top has been studied in [4], [6], [11], [22]. The present analysis is based upon the Energy-Casimir method [12], [21]. We give a necessary and sufficient condition for stability for the system of equations in complex form (8) and (9). The procedure follows closely [22].

The linearization of the nonlinear top equations (8) and (9) about the equilibrium $\omega=$ $\eta=0$, corresponding to the sleeping motion, is given by

$$
\left[\begin{array}{c}
\dot{\omega} \\
\dot{\eta}
\end{array}\right]=\left[\begin{array}{cc}
i(b-\Omega) & c \\
1 / 2 & -i \Omega
\end{array}\right]\left[\begin{array}{c}
\omega \\
\eta
\end{array}\right] .
$$

As can be easily calculated, the eigenvalues of the system (14) are

$$
\lambda_{1,2}=i \frac{b-2 \Omega}{2} \pm \frac{1}{2} \sqrt{-b^{2}+2 c} .
$$


Obviously, if $b^{2}<2 c$, then system (14) has eigenvalues in the open right half plane, which corresponds to instability of the linearized equation (14), and thus instability of the original nonlinear system (8) and (9). When $b^{2} \geq 2 c$, (14) has eigenvalues on the imaginary axis. No conclusion can be drawn for the stability of the original nonlinear system from its linearization in this case. We therefore resort to Lyapunov function theory in order to resolve the stability question of the system (8) and (9) for the case when $b^{2} \geq 2 c$. Specifically, we use the Energy-Casimir method which allows to draw stability conclusions about conservative mechanical systems when certain independent integrals of the motion (Casimirs) are known [12], [21]. According to this method, we augment the energy of the system with the Casimirs of the motion and we check for critical points of this quantity. Definiteness of the second variation at the critical points of this augmented quantity is then sufficient to prove nonlinear (Lyapunov) stability. We will not elaborate more on this issue since similar results were derived in [22]. Suffice it to say that the quantity

$$
\begin{aligned}
V(\omega, \eta) & \triangleq H(\omega, \eta)-H(0,0) \\
& =|\omega|^{2}+c \frac{1-|\eta|^{2}}{1+|\eta|^{2}}-b\left[\frac{2}{1+|\eta|^{2}} \operatorname{Im}(\omega \bar{\eta})+b \frac{1-|\eta|^{2}}{1+|\eta|^{2}}\right]-c+b^{2}
\end{aligned}
$$

is a Lyapunov function for the system (8) and (9), where

$$
H(\omega, \eta) \triangleq h_{c 1}(\omega, \eta)-b h_{c 2}(\omega, \eta)
$$

is constant under the flow of the system. This is true, because the following two expressions remain constant under the flow of the nonlinear system (8) and (9)

$$
\begin{aligned}
& h_{c 1}(\omega, \eta) \triangleq|\omega|^{2}+c \frac{1-|\eta|^{2}}{1+|\eta|^{2}} \\
& h_{c 2}(\omega, \eta) \triangleq \frac{2}{1+|\eta|^{2}} \operatorname{Im}(\omega \bar{\eta})+b \frac{1-|\eta|^{2}}{1+|\eta|^{2}}
\end{aligned}
$$

where $\operatorname{Im}(\cdot)$ denotes the imaginary part of a complex number. Actually, the first equation represents the total energy of the system, while the second equation represents the angular momentum along the inertial $Z$-axis.

It can be seen that if $b^{2} \geq 2 c$, then $V(\omega, \eta)>0$ for all $\omega, \eta \in \mathbb{C}$ although its Hessian is only positive semi-definite. Next, recall that if $b^{2}<2 c$, then the sleeping motion is unstable. Therefore we conclude that the sleeping motion of the (uncontrolled) top is Lyapunov stable if and only if $b^{2} \geq 2 c[6],[22]$.

\section{Feedback Stabilization with Two Torque Inputs}

\subsection{Complex Formulation}

We consider the controlled top problem in which two torque actuators $u_{1}, u_{2}$ along two transverse principal axes perpendicular to the symmetry axis are applied to the top; that is, 
the equations (3) are now given by

$$
\begin{aligned}
& \dot{\omega}_{1}=-(b-\Omega) \omega_{2}+\frac{c}{2} \gamma_{2}+u_{1} \\
& \dot{\omega}_{2}=(b-\Omega) \omega_{1}-\frac{c}{2} \gamma_{1}+u_{2}
\end{aligned}
$$

Defining the complex control variable $u_{c} \triangleq u_{1}+i u_{2}$, equations (20) and (9) yield

$$
\begin{aligned}
& \dot{\omega}=i(b-\Omega) \omega+\frac{c \eta}{1+|\eta|^{2}}+u_{c} \\
& \dot{\eta}=-i \Omega \eta+\frac{\omega}{2}+\frac{\bar{\omega}}{2} \eta^{2} .
\end{aligned}
$$

The control strategy employed in this subsection is based on the results of [15] and [17]. In [15], [17], globally asymptotically stabilizing control laws were derived for the motion of a symmetric spinning rigid body in space, using two control actuators. Specifically, stabilizing control laws were constructed which achieve global asymptotic stabilization of a symmetric rigid body about its symmetry axis. The control laws of [15] and [17] were based on the new complex formulation of the kinematics (6) and some well-known results for the stabilization of systems in cascade form [1], [13]. These control laws were extended in [16] and [18] in order to achieve complete reorientation from arbitrary initial conditions, utilizing the construction of the zero output dynamics manifold, with respect to an appropriately chosen system output. In [18] it was also shown that the same output for the system kinematics, when considered as a new coordinate, complements the stereographic coordinates in a natural way, providing a new parameterization of the rotation group of orthogonal matrices (the configuration space of the rotational motion).

Note that since the linearization of (21) and (22) is controllable, linear control laws based upon the linearization of these equations will, in general, only locally asymptotically stabilize the top to its sleeping motion, corresponding to the zero equilibrium $\omega=\eta=0$ of equations (8) and (9). However, since we are interested in global asymptotic stabilization we thus resort to Lyapunov function theory to construct globally asymptotically stabilizing control laws for the system (21) and (22).

Redefining the new control

$$
v \triangleq \frac{c \eta}{1+|\eta|^{2}}+u_{c}
$$

equations (21), (22) yield a system in cascade form

$$
\begin{aligned}
\dot{\omega} & =i(b-\Omega) \omega+v \\
\dot{\eta} & =-i \Omega \eta+\frac{\omega}{2}+\frac{\bar{\omega}}{2} \eta^{2} .
\end{aligned}
$$

Control laws for the system of equations (24) have been obtained in [15], [17] and can be summarized as follows. 
THEOREM 4.1 ([15]) The choice of the feedback control law

$$
v=-i(b-\Omega) \omega+\kappa\left(i \Omega \eta-\frac{\omega}{2}-\frac{\bar{\omega}}{2} \eta^{2}\right)-\alpha(\omega+\kappa \eta)
$$

with $\kappa>0$ and $\alpha>0$, globally asymptotically stabilizes system (24).

THEOREM 4.2 ([15]) The choice of the feedback control law

$$
v=-i(b-\Omega) \omega+\kappa\left(i \Omega \eta-\frac{\omega}{2}-\frac{\bar{\omega}}{2} \eta^{2}\right)-\alpha(\omega+\kappa \eta)-\eta\left(1+|\eta|^{2}\right)
$$

with $\kappa>0$ and $\alpha>0$, globally exponentially stabilizes system (24) with rate of decay $\beta / 2$, where $\beta=\min \{2 \alpha, \kappa\}$.

THEOREM 4.3 ([17]) The choice of the linear feedback control law

$$
v=-\kappa_{1} \omega-\kappa_{2} \eta
$$

with $\kappa_{1}>0$ and $\kappa_{2}>0$, globally asymptotically stabilizes the system (24).

The proofs of these theorems are shown by construction of appropriate Lyapunov functions for the corresponding closed loop systems and can be found in [15], [17].

Using the previous results and equation (23) we have the following globally asymptotically stabilizing control laws for the motion of a spinning top about its symmetry axis.

COROLLARY 4.1 The choice of the feedback control law

$$
u_{c}=-i(b-\Omega) \omega-\frac{c \eta}{1+|\eta|^{2}}+\kappa\left(i \Omega \eta-\frac{\omega}{2}-\frac{\bar{\omega}}{2} \eta^{2}\right)-\alpha(\omega+\kappa \eta)
$$

with $\kappa>0$ and $\alpha>0$, globally asymptotically stabilizes system (21)-(22).

COROLLARY 4.2 The choice of the feedback control law

$$
u_{c}=-i(b-\Omega) \omega-\frac{c \eta}{1+|\eta|^{2}}+\kappa\left(i \Omega \eta-\frac{\omega}{2}-\frac{\bar{\omega}}{2} \eta^{2}\right)-\alpha(\omega+\kappa \eta)-\eta\left(1+|\eta|^{2}\right)
$$

with $\kappa>0$ and $\alpha>0$, globally exponentially stabilizes system the (21)-(22) with rate of decay $\beta / 2$, where $\beta=\min \{2 \alpha, \kappa\}$. 
COROLLARY 4.3 The choice of the feedback control law

$$
u_{c}=-\kappa_{1} \omega-\kappa_{2} \eta-\frac{c \eta}{1+|\eta|^{2}}
$$

with $\kappa_{1}>0$ and $\kappa_{2}>0$, globally asymptotically stabilizes the system (21)-(22).

These control laws are obtained by adding an extra term in the control laws of Theorems 4.1-4.3 in order to cancel the gravitational force, as in (23). Since this obvious modification of the control laws (25), (26) and (27) depends on the exact cancellation of the gravity term, the problem of robustness of the stabilizing controls of Corollaries 4.1-4.3 need to be addressed before implementation of these results. However, since the proof of the global asymptotic stability of the corresponding closed-loop systems was demonstrated using Lyapunov functions, it is in general not very difficult to robustify the control laws (28), (29) and (30), since any such uncertainty enters the system in the same way as the control input (i.e., it is "matched") [10].

Next we present a general theory of stabilization of the equations (21) and (22) of the controlled spinning top based on Hamilton-Jacobi-Bellman (HJB) theory with zero dynamics [2], [19], [20].

\subsection{Hamilton-Jacobi-Bellman Theory with Zero Dynamics}

We use again the new kinematic equation (6) from the stereographic projection of Poisson's equations, but for convenience (and to be consistent with the standard notation in the literature of nonlinear control theory), we expand equations (21) and (22) into their real and imaginary parts. Letting $x_{1}=\omega_{1}, x_{2}=\omega_{2}, x_{3}=\eta_{1}$ and $x_{4}=\eta_{2}$, and decomposing (21) and (22) into their real and imaginary components, the equations can be written in the familiar form

$$
\dot{x}=f(x)+g(x) u=f(x)+g_{1}(x) u_{1}+g_{2}(x) u_{2}
$$

where

$$
f(x) \triangleq\left[\begin{array}{c}
-(b-\Omega) x_{2}+c x_{3} /\left(1+x_{3}^{2}+x_{4}^{2}\right) \\
(b-\Omega) x_{1}+c x_{4} /\left(1+x_{3}^{2}+x_{4}^{2}\right) \\
\Omega x_{4}+x_{2} x_{3} x_{4}+x_{1}\left(1+x_{3}^{2}-x_{4}^{2}\right) / 2 \\
-\Omega x_{3}+x_{1} x_{3} x_{4}+x_{2}\left(1-x_{3}^{2}+x_{4}^{2}\right) / 2
\end{array}\right], \quad g(x) \triangleq\left[\begin{array}{ll}
1 & 0 \\
0 & 1 \\
0 & 0 \\
0 & 0
\end{array}\right]
$$

where $x=\operatorname{col}\left(x_{1}, x_{2}, x_{3}, x_{4}\right) \in \mathbb{R}^{4}, g_{1}(x), g_{2}(x)$ are the column vectors of $g(x)$ and $u=\operatorname{col}\left(u_{1}, u_{2}\right) \in \mathbb{R}^{2}$. Clearly, $f, g_{1}$ and $g_{2}$ are $\mathcal{C}^{\infty}$ vector fields and $f(0)=0$. Below $\operatorname{ad}_{f} g$ denotes, as usual, the adjoint operation between the two vector fields $f$ and $g$ defined by [9]

$$
\operatorname{ad}_{f} g=\frac{\partial g}{\partial x} f(x)-\frac{\partial f}{\partial x} g(x)
$$


First we note that the system (32) is not globally feedback linearizable [3], [8]. The determinant of the distribution $\Delta_{1}(x) \triangleq \operatorname{span}\left\{g_{1}(x), g_{2}(x), \operatorname{ad}_{f} g_{1}(x), \operatorname{ad}_{f} g_{2}(x)\right\}$ is $\left|\Delta_{1}(x)\right|=$ $\frac{1}{4}\left(1-x_{3}^{2}-x_{4}^{2}\right)\left(1+x_{3}^{2}+x_{4}^{2}\right)$ and thus the distribution $\Delta_{1}(x)$ looses rank at $|\eta|=1$, i.e., when the tilt angle $\Theta$ is $\pm \pi / 2$. Therefore, the condition (ii) of Theorem 1 in [3] is not satisfied, since $\operatorname{dim} \Delta_{1}(x) \neq 4$ for all $x \in \mathbb{R}^{4}$. The above observation shows that it is not possible to synthesize a globally stabilizing control law for (32) using the feedback linearization approach.

The basic ingredients of the HJB theory with zero dynamics [2], [19], [20] are given next. Consider a nonlinear controlled system which is affine in the control of the form

$$
\dot{x}=f(x)+g(x) u=f(x)+g_{1}(x) u_{1}+\cdots+g_{m}(x) u_{m}
$$

where $x \in \mathbb{R}^{n}, u=\operatorname{col}\left(u_{1}, \ldots, u_{m}\right) \in \mathbb{R}^{m}$ and $g_{1}(x), \ldots, g_{m}(x)$ are the column vectors of $g(x)$. We assume $f$ and $g_{1}, \ldots, g_{m}$ are sufficiently smooth and, without loss of generality, we assume that the origin is an equilibrium state of the uncontrolled system, namely, $f(0)=0$. In order to apply the HJB theory with zero dynamics, we define an artificial output function

$$
y=h(x)
$$

where $y \in \mathbb{R}^{m}$ and $h(x)=\operatorname{col}\left(h_{1}(x), h_{2}(x), \ldots, h_{m}(x)\right)$. For the system (34), (35), consider the performance functional

$$
J\left(x_{0}, u(\cdot)\right) \triangleq \int_{0}^{\infty} L(x(t), u(t)) \mathrm{d} t
$$

where

$$
L(x, u) \triangleq L_{1}(x)+L_{2}(x) u+u^{\mathrm{T}} R u
$$

and $L_{1}: \mathbb{R}^{n} \rightarrow \mathbb{R}, L_{2}: \mathbb{R}^{n} \rightarrow \mathbb{R}^{1 \times m}$ with $L_{2}(0)=0$, and $R \in \mathbb{R}^{m \times m}$ is a positive-definite matrix. The superscript $\mathrm{T}$ denotes, as usual, the transpose.

The following definitions can be found in [9].

\section{Definitions.}

(i) The zero dynamics of the nonlinear system (34), (35) are the dynamics of this system subject to the constraint that the output $y(t)$ be identically zero for all $t \geq 0$.

(ii) The system (34), (35) is said to be minimum phase if its zero dynamics are asymptotically stable.

Let $\mathrm{L}_{f} h(x) \triangleq \nabla h(x) \cdot f(x)$ denote the Lie derivative of a function $h$ along the vector field $f$. We recall the following lemma from [2] for minimum phase systems with relative degree $\{1,1, \ldots, 1\}$ [9], which implies that the $m \times m$ matrix

$$
\mathrm{L}_{g} h(x) \triangleq\left[\begin{array}{ccc}
\mathrm{L}_{g_{1}} h_{1}(x) & \cdots & \mathrm{L}_{g_{m}} h_{1}(x) \\
\vdots & \ddots & \vdots \\
\mathrm{L}_{g_{1}} h_{m}(x) & \cdots & \mathrm{L}_{g_{m}} h_{m}(x)
\end{array}\right]
$$


is nonsingular for all $x \in \mathbb{R}^{n}$; see also [20]. Recall that a vector field $f$ is said to be complete if the flow of $f$ is defined for all times $t \in \mathbb{R}[9]$.

LEMMA 4.1 Assume that the nonlinear system (34), (35) is minimum phase with relative degree $\{1,1, \ldots, 1\}$. If the vector field $g\left(\mathrm{~L}_{g} h\right)^{-1}$ is complete, then there exists a global diffeomorphism $\theta: \mathbb{R}^{n} \rightarrow \mathbb{R}^{n}$, a $\mathcal{C}^{\infty}$ function $f_{0}: \mathbb{R}^{n-m} \rightarrow \mathbb{R}^{n-m}$, and a $\mathcal{C}^{\infty}$ function $r: \mathbb{R}^{n-m} \times \mathbb{R}^{m} \rightarrow \mathbb{R}^{(n-m) \times m}$ such that, in the coordinates

$$
\left[\begin{array}{l}
z \\
y
\end{array}\right] \triangleq \theta(x)
$$

the differential equation (34) is equivalent to the normal form

$$
\left[\begin{array}{c}
\dot{z} \\
\dot{y}
\end{array}\right]=\left[\begin{array}{c}
f_{0}(z)+r(z, y) y \\
\mathrm{~L}_{f} h(x)
\end{array}\right]+\left[\begin{array}{c}
0 \\
\mathrm{~L}_{g} h(x)
\end{array}\right] u .
$$

The next theorem gives the main result for optimal nonlinear feedback of minimum phase systems with relative degree $\{1,1, \ldots, 1\}$. The optimality of the feedback control law is guaranteed through the Hamilton-Jacobi-Bellman equation. The performance functional is assumed to include a nonquadratic state weighting and a quadratic control weighting.

THEOREM 4.4 ([20]) Consider the nonlinear system defined by equations (34), (35). Assume that the system is minimum phase with relative degree $\{1,1, \ldots, 1\}$ and the vector field $g\left(\mathrm{~L}_{g} h\right)^{-1}$ is complete. Furthermore, let $P_{0} \in \mathbb{R}^{m \times m}$ and $R \in \mathbb{R}^{m \times m}$ be positive definite and let $V_{0}: \mathbb{R}^{n-m} \rightarrow \mathbb{R}$ be a $\mathcal{C}^{1}$ positive definite function such that $D V_{0}(z) f_{0}(z)<0$, for $z \in \mathbb{R}^{n-m}, z \neq 0$. Then define

$$
\begin{aligned}
& L_{2}^{\mathrm{T}}(x)=R\left(\mathrm{~L}_{g} h\right)^{-1}\left[P_{0}^{-1} r^{\mathrm{T}}(z, y) D V_{0}(z)^{\mathrm{T}}+2 \mathrm{~L}_{f} h\right] \\
& V(x)=V_{0}(z)+y^{\mathrm{T}} P_{0} y
\end{aligned}
$$

where $z, y$ and $r(z, y)$ are defined in Lemma 4.1. Then $V(x)$ is a Lyapunov function for the closed-loop system with the control law

$$
\phi(x)=-\frac{1}{2}\left[\mathrm{~L}_{g} h(x)\right]^{-1}\left[P_{0}^{-1} r^{\mathrm{T}}(z, y) D V_{0}(z)^{\mathrm{T}}+2 \mathrm{~L}_{f} h(x)\right]-R^{-1}\left[\mathrm{~L}_{g} h(x)\right]^{\mathrm{T}} P_{0} h(x)
$$

which globally asymptotically stabilizes (34) and minimizes $J\left(x_{0}, u(\cdot)\right)$ in the sense that

$$
J\left(x_{0}, \phi(x(\cdot))\right)=\min _{u(\cdot) \in \mathcal{S}\left(x_{0}\right)} J\left(x_{0}, u(\cdot)\right)=V\left(x_{0}\right), \quad \text { for all } x_{0} \in \mathbb{R}^{n},
$$

where $J\left(x_{0}, u(\cdot)\right)$ is defined in (36)-(37), $\mathcal{S}\left(x_{0}\right)$ is the set of asymptotically stabilizing control laws, and

$$
L_{1}(x)=\phi^{\mathrm{T}}(x) R \phi(x)-\mathrm{L}_{f} V(x), \quad x \in \mathbb{R}^{n} .
$$


The performance integrand corresponding to the optimal control law (43) is [20]

$$
\begin{aligned}
L(x, u)= & \left\{u+\frac{1}{2}\left(\mathrm{~L}_{g} h\right)^{-1}\left[P_{0}^{-1} r^{\mathrm{T}}(z, y) D V_{0}(z)^{\mathrm{T}}+2 \mathrm{~L}_{f} h\right]\right\}^{\mathrm{T}} R \\
& \times\left\{u+\frac{1}{2}\left(\mathrm{~L}_{g} h\right)^{-1}\left[P_{0}^{-1} r^{\mathrm{T}}(z, y) D V_{0}(z)^{\mathrm{T}}+2 \mathrm{~L}_{f} h\right]\right\} \\
& -D V_{0}(z) f_{0}(z)+h^{\mathrm{T}}(x) P_{0}\left(\mathrm{~L}_{g} h\right) R^{-1}\left(\mathrm{~L}_{g} h\right)^{\mathrm{T}} P_{0} h(x)
\end{aligned}
$$

which is nonnegative for all $x \in \mathbb{R}^{n}$ and $u \in \mathbb{R}^{m}$. In the above expressions $D V_{0}(z)$ denotes the Jacobian of $V_{0}$ with respect to $z$.

\subsection{The Spinning Top}

In the statement of Theorem 4.4 is implicit the fact that there exists an output for the nonlinear system with respect to which the overall system has relative degree $\{1,1, \ldots, 1\}$. Therefore, the existence of such an output is crucial for the Theorem 4.4 to be applicable. Note that as in the case of feedback linearizable systems the desired output may not be necessarily the given output of the system. Instead it is often up to the control designer to choose such an output in order to achieve relative degree one with respect to all output channels. This is not necessarily a trivial task, and a judicious choice may facilitate the analysis and the control design.

For the controlled spinning top problem, let the output function be

$$
y=h(x)=\left[\begin{array}{l}
h_{1}(x) \\
h_{2}(x)
\end{array}\right] \triangleq\left[\begin{array}{l}
x_{1}+k_{1} x_{3} \\
x_{2}+k_{2} x_{4}
\end{array}\right]
$$

where $k_{1}>0$ and $k_{2}>0$. Defining $z=\operatorname{col}\left(z_{1}, z_{2}\right) \triangleq \operatorname{col}\left(x_{3}, x_{4}\right)$, we have

$$
\dot{z}=f_{0}(z)+r(z, y) y
$$

where

$$
\begin{aligned}
& f_{0}(z) \triangleq\left[\begin{array}{cc}
-\frac{1}{2} k_{1} z_{1}+\Omega z_{2}-\frac{1}{2} k_{1} z_{1}^{3}-\left(k_{2}-\frac{k_{1}}{2}\right) z_{1} z_{2}^{2} \\
-\frac{1}{2} k_{2} z_{2}-\Omega z_{1}-\frac{1}{2} k_{2} z_{2}^{3}-\left(k_{1}-\frac{k_{2}}{2}\right) z_{1}^{2} z_{2}
\end{array}\right] \\
& r(z, y)=\left[\begin{array}{cc}
\frac{1}{2}\left(1+z_{1}^{2}-z_{2}^{2}\right) & z_{1} z_{2} \\
z_{1} z_{2} & \frac{1}{2}\left(1-z_{1}^{2}+z_{2}^{2}\right)
\end{array}\right] .
\end{aligned}
$$

It can be shown that the zero dynamics corresponding to $\dot{z}=f_{0}(z)$ is globally asymptotically stable and the corresponding Lyapunov function is

$$
V_{0}(z)=p_{3}\left(z_{1}^{2}+z_{2}^{2}\right), \quad p_{3}>0 .
$$

Furthermore, $\mathrm{L}_{g} h(x)=\mathrm{I}_{2}$, where $\mathrm{I}_{2}$ is the $2 \times 2$ identity matrix. Hence (32) and (47) form 
a minimum phase system with relative degree $\{1,1\}$. Next, by taking

$$
P_{0}=\left[\begin{array}{cc}
p_{1} & 0 \\
0 & p_{2}
\end{array}\right], \quad R=\left[\begin{array}{cc}
r_{1} & 0 \\
0 & r_{2}
\end{array}\right]
$$

with $p_{1}, p_{2}>0$ and $r_{1}, r_{2}>0$, and applying Theorem 4.4 to the system (32) and (47) the optimal control law $\phi(x)=\operatorname{col}\left(\phi_{1}(x), \phi_{2}(x)\right)$ is computed from (43) to be

$$
\begin{aligned}
\phi_{1}(x)= & (b-\Omega) x_{2}-c x_{3} /\left(1+x_{3}^{2}+x_{4}^{2}\right) \\
& -k_{1}\left[\Omega x_{4}+x_{2} x_{3} x_{4}+\frac{x_{1}}{2}\left(1+x_{3}^{2}-x_{4}^{2}\right)\right] \\
& -\left(p_{3} x_{3} / 2 p_{1}\right)\left(1+x_{3}^{2}+x_{4}^{2}\right)-\left(p_{1} / r_{1}\right)\left(x_{1}+k_{1} x_{3}\right) \\
\phi_{2}(x)= & -(b-\Omega) x_{1}-c x_{4} /\left(1+x_{3}^{2}+x_{4}^{2}\right) \\
& -k_{2}\left[-\Omega x_{3}+x_{1} x_{3} x_{4}+\frac{x_{2}}{2}\left(1-x_{3}^{2}+x_{4}^{2}\right)\right] \\
& -\left(p_{3} x_{4} / 2 p_{2}\right)\left(1+x_{3}^{2}+x_{4}^{2}\right)-\left(p_{2} / r_{2}\right)\left(x_{2}+k_{2} x_{4}\right) .
\end{aligned}
$$

The Lyapunov function that guarantees asymptotic stability of the closed-loop system with the control law (53) is given from (42)

$$
V(x)=p_{3}\left(x_{3}^{2}+x_{4}^{2}\right)+p_{1}\left(x_{1}+k_{1} x_{3}\right)^{2}+p_{2}\left(x_{2}+k_{2} x_{4}\right)^{2}
$$

while the performance integrand (46) is

$$
\begin{aligned}
L(x, u)= & {\left[u+\frac{1}{2} R^{-1} L_{2}^{\mathrm{T}}(x)\right]^{\mathrm{T}} R\left[u+\frac{1}{2} R^{-1} L_{2}^{\mathrm{T}}(x)\right] } \\
& +p_{3}\left[k_{1} x_{3}^{2}\left(1+x_{3}^{2}\right)+k_{2} x_{4}^{2}\left(1+x_{4}^{2}\right)+\left(k_{1}+k_{2}\right) x_{3}^{2} x_{4}^{2}\right] \\
& +\frac{p_{1}^{2}}{r_{1}}\left(x_{1}+k_{1} x_{3}\right)^{2}+\frac{p_{2}^{2}}{r_{2}}\left(x_{2}+k_{2} x_{4}\right)^{2}
\end{aligned}
$$

where

$$
L_{2}^{\mathrm{T}}(x)=\left[\begin{array}{c}
r_{1}\left\{p_{3} x_{3}\left(1+x_{3}^{2}+x_{4}^{2}\right) / p_{1}-2(b-\Omega) x_{2}+2 c x_{3} /\left(1+x_{3}^{2}+x_{4}^{2}\right)\right. \\
\left.+2 k_{1}\left[\Omega x_{4}+x_{2} x_{3} x_{4}+x_{1}\left(1+x_{3}^{2}-x_{4}^{2}\right) / 2\right]\right\} \\
r_{2}\left\{p_{3} x_{4}\left(1+x_{3}^{2}+x_{4}^{2}\right) / p_{2}+2(b-\Omega) x_{1}+2 c x_{4} /\left(1+x_{3}^{2}+x_{4}^{2}\right)\right. \\
\left.+2 k_{2}\left[-\Omega x_{3}+x_{1} x_{3} x_{4}+x_{2}\left(1-x_{3}^{2}+x_{4}^{2}\right) / 2\right]\right\}
\end{array}\right] .
$$

Equation (53) provides a family of feedback stabilizing control laws for the system (32), which are optimal with respect to the performance functional (55). This 7-parameter family $\left(k_{1}, k_{2}, r_{1}, r_{2}, p_{1}, p_{2}, p_{3}\right)$ allows for great flexibility in the design of optimal feedback control laws for the spinning top. It can be easily checked that by taking $k_{1}=k_{2}=\kappa, p_{1} / r_{1}=$ $p_{2} / r_{2}=\alpha$ and $p_{3} / p_{1}=p_{3} / p_{2}=2$, the control law (53) reduces to (29). Notice, however, that the control laws (28) and (30) cannot be derived form (53) by any admissible choice of the parameters.

Remark. It should be pointed out that the control laws obtained above are globally asymptotically stabilizing for all $x \in \mathbb{R}^{4}$. Physically, this implies global asymptotic stability for 
the closed loop system from all initial configurations, except in the case when $\eta=\infty$. This case corresponds to direction cosines $\left(\gamma_{1}, \gamma_{2}, \gamma_{3}\right)=(0,0,-1)$ in equation (5), that is, the top symmetry axis is along the downward direction. Therefore, global stability here implies stability from all initial conditions except the initial condition corresponding to this singular "upside-down" configuration. (Note that by the global stabilizing nature of the control laws of the system in $(\omega, \eta)$ coordinates, one has that $\eta(t)<\infty$ for all $t \geq 0$ as long as $\eta(0) \neq \infty$.) If the top is initially upside down, then one can apply an arbitrary input to drive the top to any nonsingular orientation. The stabilizing control laws obtained above can then be applied from this new orientation. Thus, the top can be globally asymptotically stabilized to the sleeping motion, including the singular one.

\section{Numerical Examples}

In this section we apply the control laws obtained in Section 4 to stabilize the spinning top to the sleeping motion. We assume that the top parameters are $J_{1}=\ell=1, m g=3$ and $J_{3}=0.2$. If $\Omega=1$ then $b=0.2, c=6$, which corresponds to a unstable top. If the initial conditions are $x(0)=(0,0,0.01,0.01)^{\mathrm{T}}$ which implies initially the slowly spinning top has zero transverse angular velocity with tilt angle $\Theta=1.62 \mathrm{deg}$. Obviously, without external control inputs the top will fall toward the downward position. To demonstrate the effect of the control laws, we apply the control laws (53) at $t=3.1 \mathrm{sec}$ when the tilt angle is about $150 \mathrm{deg}$. Figures 1-3 show the time history of the tilt angle $\Theta$, the states $x_{3}, x_{4}$ and the control effort $u_{1}, u_{2}$, respectively. It is seen from Figures 1 and 2 that before the controls are applied $(t<3.1 \mathrm{sec})$, the tilt angle $\Theta$ grows rapidly and the states $x_{3}, x_{4}$ decrease rapidly to large negative values, which correspond to the upside down configuration, and after the controls are applied the tilt angle $\Theta$ and the states $x_{3}, x_{4}$ are driven to zero asymptotically which correspond to the sleeping motion of the top.

As another example, we consider a stable top which is initially under steady precession. The specifications of the top are $J_{1}=\ell=1, m g=3$ and $J_{3}=4 / 3.5$. If $\Omega=3.5$ then $b=4, c=6$. The initial conditions are $x(0)=(11.47,3.45,-.407,1.354)^{\mathrm{T}}$, which implies that the top is initially precessing with tilt angle $\Theta=109.47 \mathrm{deg}$. The control laws (53) are applied at $t=15 \mathrm{sec}$. Figures 4-6 show the tilt angle $\Theta$, the states $x_{3}, x_{4}$ and the control effort $u_{1}, u_{2}$, respectively. It is seen from Figures 4 and 5 that before the controls are applied $(t<15 \mathrm{sec})$ the tilt angle $\Theta$ is constant and the states $x_{3}, x_{4}$ are periodic functions. After application of the feedback control law the tilt angle $\Theta$ and the states $x_{3}, x_{4}$ are driven to zero asymptotically.

We note in passing that, without loss of generality, the gain and control parameters in these simulations were all taken equal to unity.

\section{Conclusions}

In this paper, the stability and stabilization of a spinning top were examined. A new formulation of the kinematics, which facilitates the design of feedback control laws is introduced. In particular, the use of stereographic coordinates is shown to be extremely 


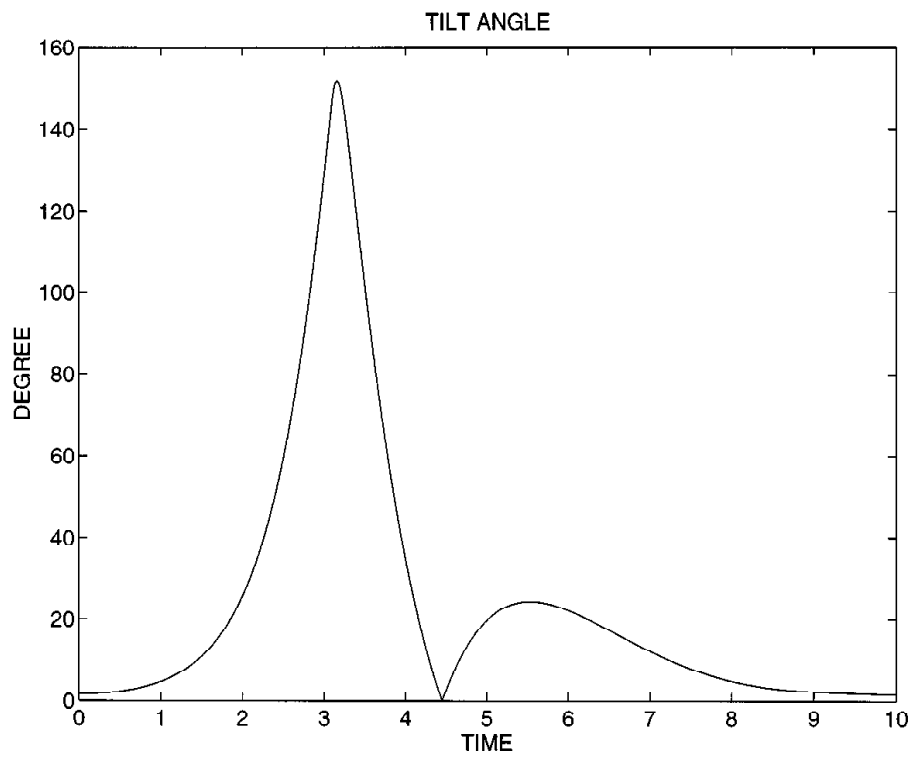

Figure 1. Tilt angle $\Theta$ vs. time.

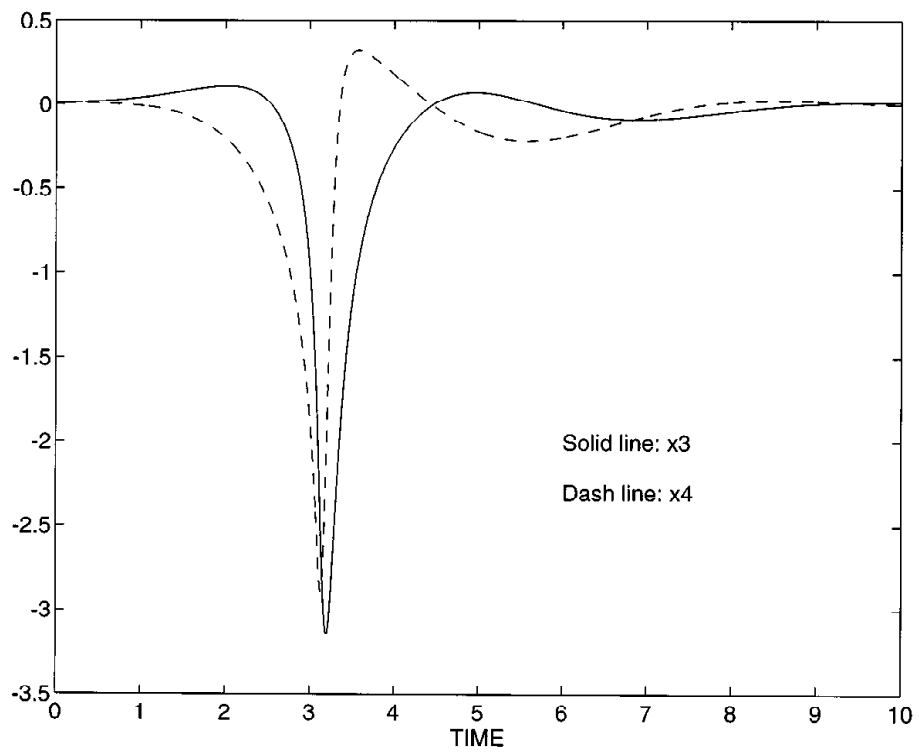

Figure 2. States $x_{3}$ and $x_{4}$ vs. time. 


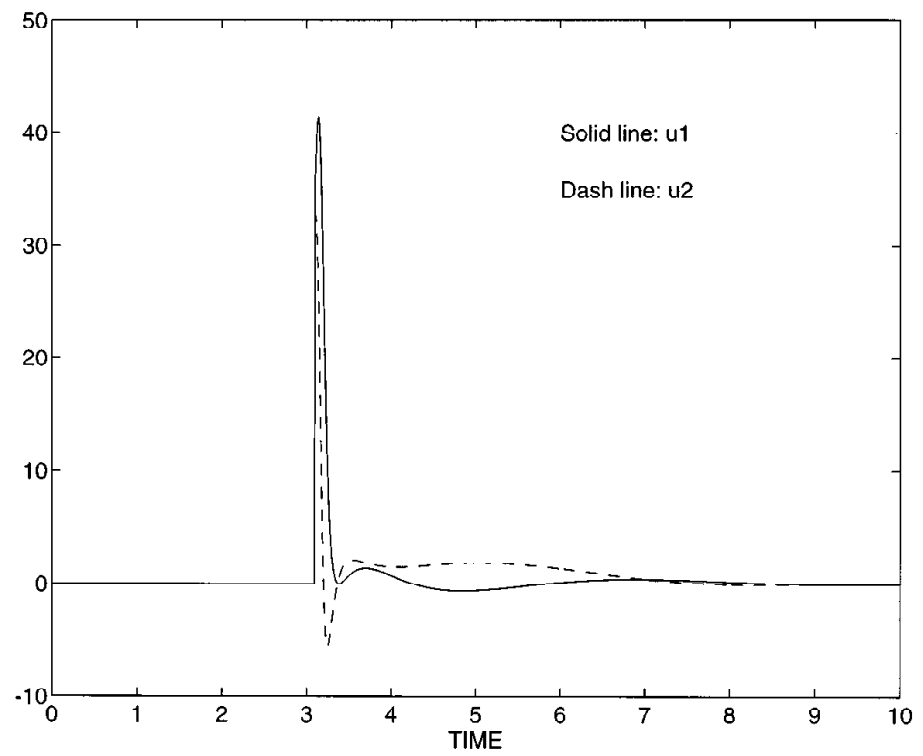

Figure 3. Control history for $u_{1}$ and $u_{2}$.

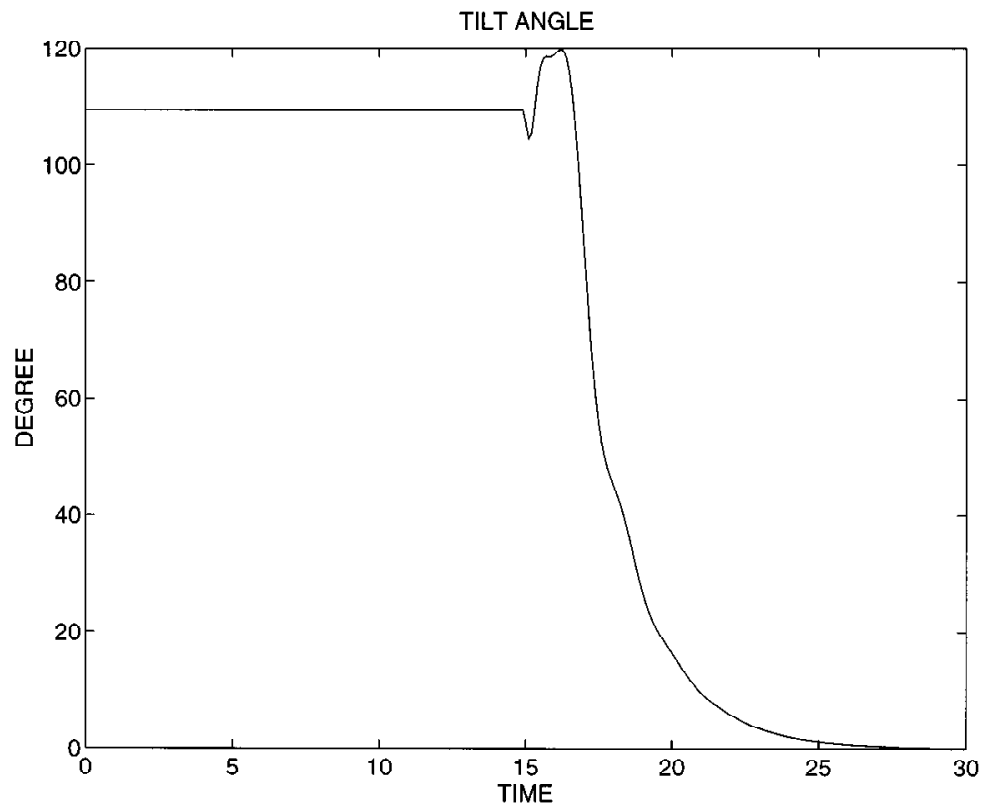

Figure 4. Tilt angle $\Theta$ vs. time. 


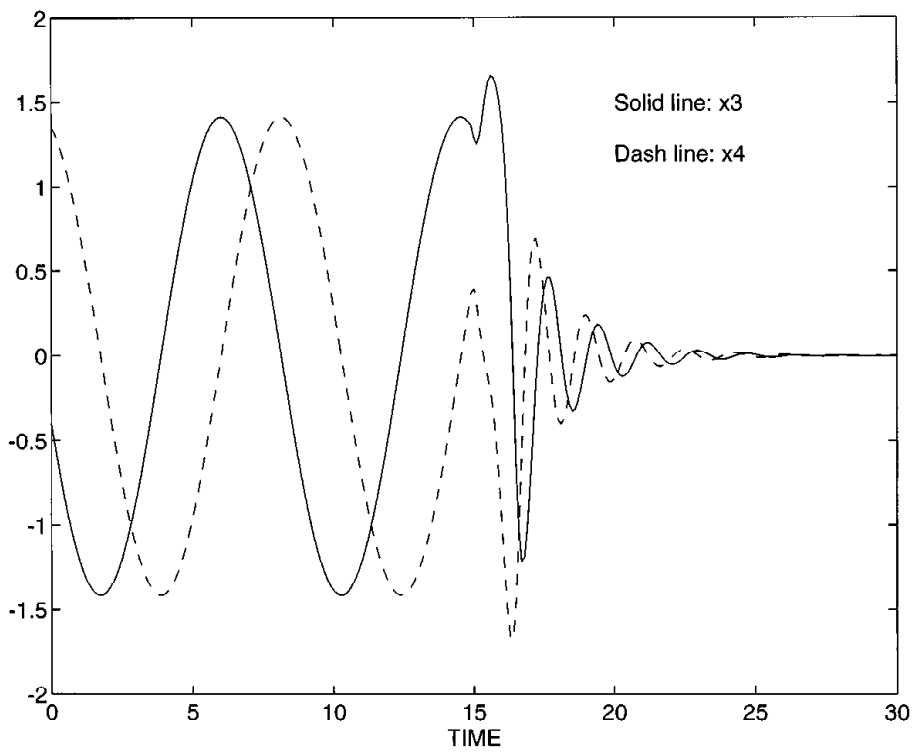

Figure 5. States $x_{3}$ and $x_{4}$ vs. time.

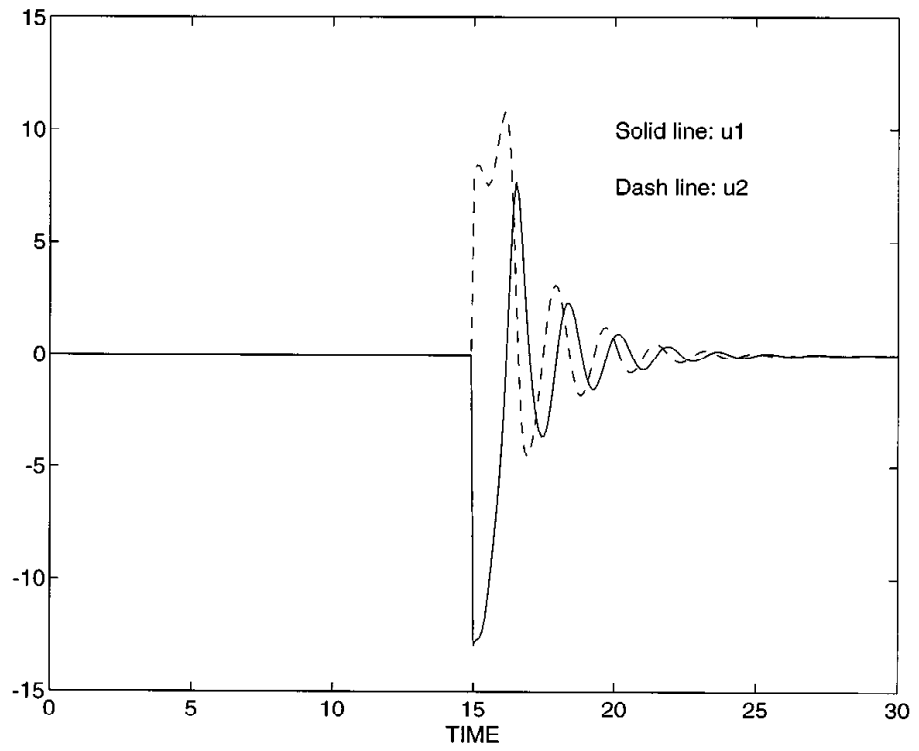

Figure 6. Control history for $u_{1}$ and $u_{2}$. 
helpful, facilitating the analysis and control design for problems in rotational dynamics. Stabilizing control laws using only two torque actuators were synthesized by employing techniques from the theory of cascade systems and from Hamilton-Jacobi-Bellman theory with zero dynamics. The final result is a methodology which leads to a construction of a family of optimal, nonlinear feedback control laws, which provide great flexibility in the control synthesis. Although the methodology is demonstrated using the spinning top example, it is believed that this theory will be helpful for a broad class of problems encountered in rotational dynamics and kinematics.

\section{Acknowledgments}

Research supported in part by the Air Force Office of Scientific Research under Grant F49620-92-J-0127.

\section{References}

1. Aeyels, D., "Remarks on the stabilizability of nonlinear systems by smooth feedback," in Perspectives in Control Theory: Proc. of the Sielpia Conference, Sielpia, Poland, Sept. 19-24, 1988, eds. B. Jakubczyk, K. Malanowski and W. Respondek, pp. 1-11, Birkhäuser: Boston,1990.

2. Byrnes, C. I. and Isidori, A., "New results and examples in nonlinear feedback stabilization," Sys. Contr. Lett., vol. 12, pp. 437-442, 1989.

3. Cheng, D. C., Tarn, T. J., and Isidori, A., "Global extended linearization of nonlinear systems via feedback," IEEE Trans. Autom Contr., vol. AC-30, pp. 808-811, 1985.

4. Chetayev, N. G., The Stability of Motion, Pergamon Press: New York, 1961.

5. Darboux, G., Lecons sur la Théorie Générale des Surfaces, vol. 1, Gauthier-Villars: Paris, 1887.

6. Ge, Z. M. and Wu, Y. J., "Another theorem for determining the definiteness of sign of functions and its applications to the stability of permanent rotations of a rigid body," Trans. of ASME, Journal of Applied Mechanics, vol. 51, pp. 430-434, 1984.

7. Greenwood, D. T., Principles of Dynamics, 2nd edition, Prentice-Hall: New Jersey, 1988.

8. Hunt, L. R., Su, R., and Meyer, G. "Design for multi-input nonlinear systems," in Differential Geometric Control Theory, Progress in Mathematics, eds. R. W. Brockett, R. S. Millman , and H. J. Sussmann, vol. 27, pp. 268-298, 1983.

9. Isidori, A., Nonlinear Control Systems, 2nd edition, Springer-Verlag: Heidelberg, 1989.

10. Khalil, H. K., Nonlinear Systems, MacMillan Publishing Co.: New York, 1992.

11. Leimanis, E., The General Problem of the Motion of Coupled Rigid Bodies about a Fixed Point, Springer Verlag: New York, 1965.

12. Rouche, N., Habets, P., and Laloy, M., Stability Theory by Lyapunov's Direct Method, Springer Verlag: New York, 1977.

13. Seibert, P., and Suarez, R., "Global stabilization of nonlinear cascade systems," Sys. Contr. Lett., vol. 14, pp. 347-352, 1990.

14. Tsiotras, P., and Longuski, J. M., "New kinematic relations for the large angle problem in rigid body attitude dynamics," Acta Astronautica, vol. 32, pp. 181-190, 1994.

15. Tsiotras, P., and Longuski, J. M., "On attitude stabilization of symmetric spacecraft with two control torques," in Proc. Amer. Contr. Conf., San Francisco, CA, pp. 46-50, 1993.

16. Tsiotras, P., Corless, M., and Longuski, J. M., "Invariant manifold techniques for attitude control of spacecraft," in Proc. Conf. Dec. Contr., San Antonio, TX, pp. 1470-1475, 1993.

17. Tsiotras, P., and Longuski, J. M., "Spin-axis stabilization of symmetric spacecraft with two control torques," Sys. Contr. Lett., vol. 23, pp. 395-402, 1994. 
18. Tsiotras, P., Corless, M., and Longuski, J. M., "A novel approach for the attitude control of an axisymmetric spacecraft subject to two control torques," Automatica, vol. 31, pp. 1099-1112, 1995.

19. Wan, C. J., and Bernstein, D. S., "A family of optimal nonlinear feedback controllers that globally stabilize angular velocity," in Proc. Conf. Dec. Contr., Tucson, AZ, pp. 1143-1148, 1992.

20. Wan, C. J., and Bernstein, D. S., "Nonlinear feedback control with global stabilization," Dynamics and Control, vol. 15, pp. 321-346, 1995.

21. Wan, C. J., Coppola, V. T., and Bernstein, D. S., "A Lyapunov function for the energy-Casimir method," in Proc. Conf. Dec. Contr., San Antonio, TX, pp. 3122-3123, 1993.

22. Wan, C. J., Coppola, V. T., and Bernstein, D. S., "Global asymptotic stabilization of the spinning top," Opt. Cont. Appl. Meth., vol. 16, pp. 189-215, 1995. 